Research Article

\section{Pathogen identification and control of sooty spot caused by Cladosporium ramotenellum, appearing on fresh easy peeler mandarins from Perú}

\section{Celia Murciano ${ }^{1 *}$, Jeniffer J Oliver-Chirito ${ }^{1,2}$ and Benito Orihuel-Iranzo ${ }^{1}$}

1Productos Citrosol S.A, Partida Alameda Parc. C, 46721, Potríes, Valencia, Spain

${ }^{2}$ Asociación de Productores de Cítricos del Perú, Av. Nicolás Arriola 314 Of. 903, La Victoria, Lima, Perú

\section{Abstract}

During the 2018 season, superficial dry and firm black spots, where sometimes an aerial mycelium developed, appeared on the rind of easy peeler mandarins causing high economic losses in fresh citrus exports from Perú. In this work, we have identified the causal agent, a species of Cladosporium not previously reported as a citrus pathogen. The pathogen was isolated from rind lesions of affected fruit and was identified by sequencing as Cladosporium ramotenellum; and fulfilment of Koch postulates was proven. This species was present on the surface of immature fruit in the groves, indicating that the infection is likely initiated before harvest. Cladosporium ramotenellum is resistant to the postharvest fungicides imazalil, pyrimethanil, and thiabendazole, but sensitive to propiconazole, prochloraz, and ortho-phenylphenol. We designed a postharvest industrial treatment to decrease the Cladosporium $\mathrm{sp}$. load on the fruit surface that limited the incidence of infection and reduced the postharvest losses caused by the fungus. Although this species is quite ubiquitous, this is the first description of $C$. ramotenellum causing decay of citrus fruit, being the symptoms of this disease similar to the ones described previously and caused by Cladosporium cladosporoides in cv. Satsuma mandarins from Japan.

\section{More Information}

*Address for Correspondence: Celia Murciano, Productos Citrosol S.A, Partida Alameda Parc. C, 46721, Potríes, Valencia, Spain, Tel: +34962800512; Email: cmurciano@citrosol.com

Submitted: May 13, 2021

Approved: June 08, 2021

Published: June 09, 2021

How to cite this article: Murciano $C$, Oliver-Chirito JJ, Orihuel-Iranzo B. Pathogen identification and control of sooty spot caused by Cladosporium ramotenellum, appearing on fresh easy peeler mandarins from Perú. J Plant Sci Phytopathol. 2021; 5: 044-052.

DOI: 10.29328/journal.jpsp.1001059

ORCiD: orcid.org/0000-0003-0035-5306

Copyright: @ 2021 Murciano C, et al. This is an open access article distributed under the Creative Commons Attribution License, which permits unrestricted use, distribution, and reproduction in any medium, provided the original work is properly cited.

Keywords: Citrus; Disease; Sooty spot; Cladosporium ramotenellum; Rot; Postharvest control; Chilling injury

Abbreviations: Cl: Chilling Injury; IMZ: imazalil; OPP: ortho-phenylphenol; PAA: Peracetic Acid; PCL: Prochloraz; PPZ: Propiconazole; PYR: Pyrimethanil; TBZ: Thiabendazole

Check for updates

OPEN ACCESS

\section{Introduction}

Fresh citrus are an important part of the human diet worldwide, and citrus shipments between continents are generally increasing (https://worldcitrusorganisation.org/ wp-content/uploads/2020/01/Citrus-Market-Trends-2019. pdf). In 2019, Perú exported 200.000tn of fresh citrus, 80\% of these were different varieties of easy peelers - Satsumas, Nadorcott, W. Murcott, Tango and others [data from the World Citrus Organization (WCO) and the Association of Citrus Producers of Peru (PROCITRUS)].

During the 2018 season, high postharvest losses occurred in some varieties of easy peelers shipped from Perú to China, Europe and United States. Losses appeared to be higher in late season shipments bound to countries where a cold quarantine is required. The losses were due mainly to circular shape superficial dry and firm black spots over the entire fruit skin without any identifiable pattern (Figure 1a-c). Lesions were not detectable at origin, when the fruit were being packed, but developed during the 15 to 45 days of refrigerated transportation and after container unloading at destination. Once transferred to higher temperatures, some fruits developed a grey-olivaceous or black mycelium on top of the black spots (Figure 1d-f). Although no maceration of the rind or flesh was noticed, the fruit could not be sold because of the highly unpleasant appearance of the rind. The cultivar most affected was W. Murcott, but there were also reports of cvs. Nadorcott and Tango mandarins with the same decay problem. A similar problem was previously described in shipped greenhouse grown Satsuma mandarins in Japan in 2006 [1]. The causal agent was identified as Cladosporium cladosporioides, and the disease was named sooty spot. 


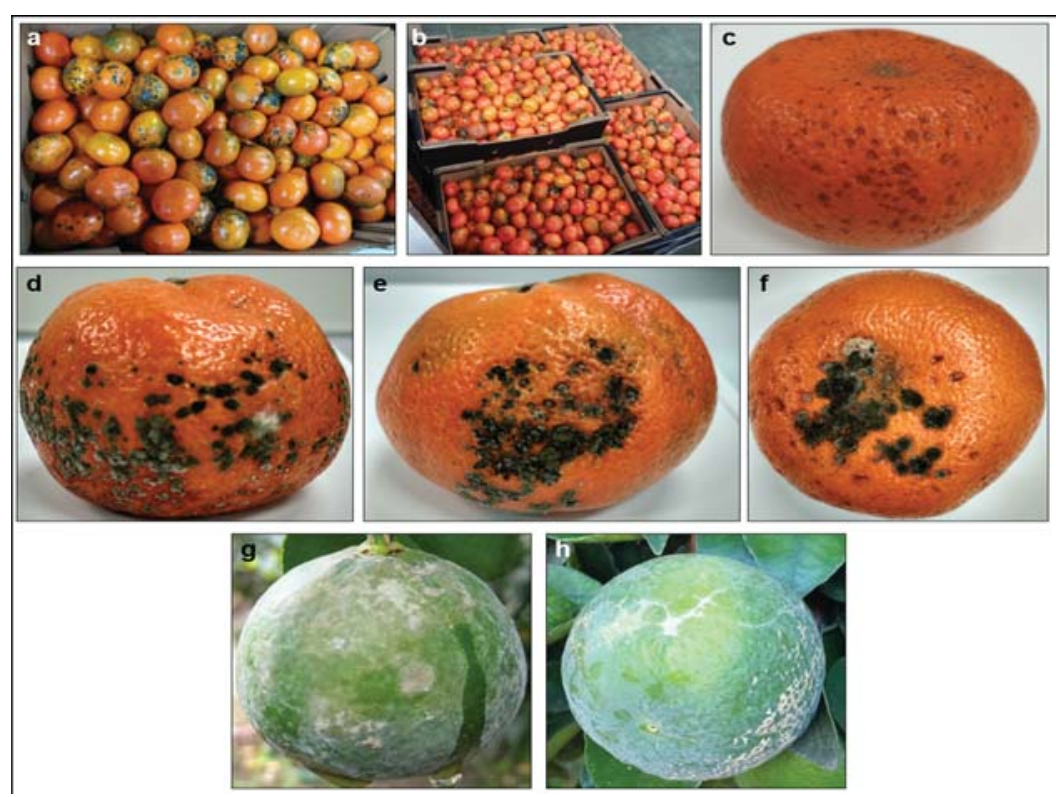

Figure 1: Representative images of fruit diseased by Cladosporium ramotenellum sooty spot. Boxes of W. Murcott mandarins affected after 15 to 22 days of cold transportation from Perú to USA (a-b). Details of W. Murcott mandarin diseased, showing the initial pitting (c), and fruits where some of the spots have developed aerial mycelium (d-f). Green immature W. Murcott mandarins before being collected to analyse the presence of Cladosporium sp. in the field $(\mathrm{g}-\mathrm{h})$.

Cladosporium species are broadly distributed and can be found on many types of substrates (plants, soil, food, organic matter, etc.) [2]. Recent phylogenetic studies recognised more than 170 Cladosporium species, belonging to three species complexes: the herbarum complex, the cladosporioides complex, and the sphaerospermum complex [2]. Cladosporium spp. can infect citrus fruit when spores are deposited onto weakened areas of the rind and germinate in storage conditions of high relative humidity. The fungus can grow at low temperatures of close to $0^{\circ} \mathrm{C}$. The decay affects mainly the rind, producing firm but flexible areas that become dark. Later, a gray-olivaceous or black mycelial growth may cover the black spots [3]. In citrus, the Cladosporium species associated with decay have been C. herbarum [4] and C. cladosporioides $[1,3]$. However, Cladosporium sp. is not a common citrus pathogen. The frequency of natural infections caused by this fungus is very low and affects mainly to fruits with injuries and rind disorders, as the infection of the fruit starts through fissures or cracks in the rind [3].

The aim of this work was to isolate the causal agent of the black spots on the rind of easy peeler mandarins exports from Perú, that caused such high economic losses during the 2018 season. To that end we have isolated, by microbiological techniques, and identified, by genomic sequencing, a Cladosporium species not previously reported as a citrus pathogen. Additionally, in order to design a successful postharvest treatment of the fruit aimed to reduce the incidence of the disease, we tested the sensitivity of the causal pathogen to the postharvest fungicides currently registered worldwide and tested different industrial application programmes with regard to the survival of the Cladosporium sp. inoculum on the fruit rind.

\section{Material and methods}

\section{Fruits}

Samples of 10 to $15 \mathrm{~W}$. Murcott mandarins were collected from two packinghouses in the Chincha region of Perú at the end of the 2018 season. Collected fruit were left in cold storage so that the damage could be investigated. These fruit had a high incidence of black spots, some of them with aerial mycelium (Figure 1c-f).

Additionally, to check the origin of the infection, green immature W. Murcott mandarins were collected from Chincha fields in December 2018 (Figure 1g,h).

\section{Chemical compounds and preparation of disinfection and fungicide solutions}

The following chemical compounds used in this study were supplied by PRODUCTOS CITROSOL S.A. (Valencia, Spain): Citrocide ${ }^{\circledR}$ Plus (peracetic acid (PAA) 15\% weight/weight $\left.(\mathrm{w} / \mathrm{w})+\mathrm{H}_{2} \mathrm{O}_{2} 23 \% \mathrm{w} / \mathrm{w}\right)$, Citrocide ${ }^{\circledR}$ PC (PAA $5 \% \mathrm{w} / \mathrm{w}+\mathrm{H}_{2} \mathrm{O}_{2}$ $23 \% \mathrm{w} / \mathrm{w}$ ), Imacide ${ }^{\circledR} 7.5$ LS (Imazalil (IMZ) $7.5 \%$ weigth/ volume (w/v)), Ortocil ${ }^{\circledR}$ (orthophenylphenol (OPP) 10\% w/v), Citropyr $^{\circledR} 40$ SC (pyrimethanil (PYR) 40\% w/v), Tiabendazol 60 (thiabendazole (TBZ) 60\% w/v), Citrocil ${ }^{\circledR}$ (IMZ 7.5\% w/v + OPP $10 \% \mathrm{w} / \mathrm{v})$, Fortisol ${ }^{\circledR} \mathrm{Ca}(14.44 \% \mathrm{w} / \mathrm{v}$ of a mix of watersoluble salts of calcium $(\mathrm{CaO})$, sodium $\left(\mathrm{Na}_{2} \mathrm{O}\right)$ and phosphorus $\left(\mathrm{P}_{2} \mathrm{O}_{5}\right)$ ), Citrosol Sunseal ${ }^{\circledR}$ UE IMAD 2 (Polyethylene + Shellac wax supplemented with IMZ $0.2 \% \mathrm{w} / \mathrm{w}$ ), Citrotec $45 \mathrm{SC}$ (TBZ $45 \% \mathrm{w} / \mathrm{v}$ ), Ortosol 6500 (OPP 28,6\% w/v).

Prochloraz (PCL; $\geq 95.0 \% \mathrm{w} / \mathrm{v}$ ) and propiconazole (PPZ; $\geq 95.0 \% \mathrm{w} / \mathrm{v}$ ) were purchased from Molekula (Molekula Ltd, Darlington, United Kingdom). 
The disinfection solution used to surface disinfect the fruit was prepared using a $0,3 \% \mathrm{v} / \mathrm{v}$ solution in water of Citrocide ${ }^{\circledR}$ Plus to achieve a final concentration of $450 \mathrm{ppm}$ PAA +690 ppm of $\mathrm{H}_{2} \mathrm{O}_{2}$.

The $400 \mathrm{ppm}$ fungicide solutions used to test the fungicide resistance or sensitivity of the isolates were prepared by diluting the above chemicals compounds in sterile water as follow: IMZ, $0.53 \% \mathrm{v} / \mathrm{v}$ Imacide $^{\circledR} 7.5 \mathrm{LS} ;$ OPP, $0.4 \% \mathrm{v} / \mathrm{v}$ Ortocil $^{\circledR}$; PCL, $0.042 \% \mathrm{v} / \mathrm{v}$ prochloraz; PPZ, $0.042 \% \mathrm{v} / \mathrm{v}$ propiconazole; PYR, $0.1 \% \mathrm{v} / \mathrm{v}$ Citropyr ${ }^{\circledR} 40 \mathrm{SC}$; and TBZ, $0.067 \% \mathrm{v} / \mathrm{v}$ Tiabendazol 60 . The concentration of the solutions was checked by High-Performance Liquid Chromatography (HPLC) before its use.

The postharvest treatments used in the packinghouse were applied as described in table 1.

\section{Pathogen isolation, identification, and determination of the inoculum on fruit}

To isolate the pathogen causing the decay, affected fruits were surface disinfected with $70 \%$ ethanol. Next, the outer layer of the rind was removed using a sterile scalpel to eliminate any potential saprophytic microorganisms growing on the diseased tissue. Pieces of albedo from the decay zone were then sampled using a sterile scalpel and placed onto Potato dextrose agar (PDA; Scharlau, Barcelona, Spain) petri dishes and incubated at $22{ }^{\circ} \mathrm{C}$ for 6 to 8 days. Pure cultures were then maintained on PDA and sub cultured every 3 to 4 weeks.

To determine the presence of Cladosporium spp. in the field, the fungal load on the surface of green immature W. Murcott mandarins was determined. For this, each fruit was weighted and then placed in a sterile plastic bag. $10 \mathrm{~mL}$ of buffered peptone water (Scharlau) amended with $0.1 \%$ Tween-20 (Wall Chemie GmbH, Kempen, Germany) was added to the bag and, after firmly closing it, the surface of the fruit was scrubbed for $1 \mathrm{~min}$. The washing liquid was recovered, and ten-fold serial dilutions were plated per duplicate onto PDA petri dishes. The fungal load was calculated as cfu/gr (colony forming units/gram) for each fruit.

Identification of the pathogen genus was initially performed based on the macroscopic colony morphology and the microscopic spore morphology.

Species identification was performed by amplification, partial Sanger sequencing and bioinformatic analysis by the company Darwin Bioprospecting (https://darwinbioprospecting.com/; Valencia Spain).

For this, the purity of the cultures was assured by light microscopy and the genomic DNA of the isolates was extracted by heat shock following the methodology described by Pascual, et al. [5].

Subsequently, three molecular markers proposed by Bensch, et al. [2] for the accurate taxonomic identification of strains belonging to the genus Cladosporium were sequenced: (i) elongation factor $1 \alpha(\mathrm{EF}-1 \alpha)$, (ii) actin (ACT), and (iii) ribosomal genes and their corresponding intergenic regions ( ITS1-5S-ITS2-28S). Amplification and sequencing of the EF-1 $\alpha$ marker was performed using the primer pair

EF1-728F (5'-CATCGAGAAGAAGTTCGAGAAGAAGG-3')and EF1986R (5'-TACTTGAAGGAAGGAACCCTTACC-3'), while for the actin gene the pair ACT-512F (5'ATGTGCA-AGGCCGGGGTTTCGC-3') and ACT-783R (5'-TACGAGTCCTTCTTCTGGCCCAT-3') was used according to Carbone and Kohn [6]. The primers V9G (5'- TTACGTCCCTGCCCTTTGTA- 3') following De Hoog and van den Ende [7] and LR5 (5'- ATCCTGAGGAGGGAAACTTC-3') as described by Vilgalys and Hester [8] were used to amplify the region from the 3 ' end of the $18 \mathrm{~S}$ ribosomal gene, the first intergenic region ITS1, the 5S ribosomal gene, the second intergenic region ITS2 and approximately the first 900 bases of the $28 \mathrm{~S}$ ribosomal gene. PCR conditions and thermal cycles used are reported in Bensch, et al. [2]. Amplification products were checked by electrophoresis on $1.2 \%(\mathrm{w} / \mathrm{v})$ agarose gels. Subsequently, the

Table 1: Postharvest treatments applied to the fruit and methods of application in an industrial packinghouse.

\begin{tabular}{|c|c|c|c|}
\hline Sample Id & Sampling Point & Postharvest Treatment & Concentrations of active Ingredients \\
\hline 1 & Field Fruit & None & - \\
\hline \multirow{3}{*}{2} & \multirow{3}{*}{ After drenching $\mathrm{A}$} & Citrocil $^{\circledR} 0.6 \%$ & 450 ppm IMZ + 600 ppm OPP \\
\hline & & Citrocide ${ }^{\circledR}$ Plus $0.1 \%$ & 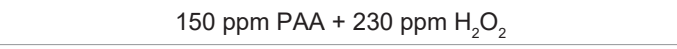 \\
\hline & & Fortisol ${ }^{\circledR} \mathrm{Ca} 1.2 \%$ & Calcium $(\mathrm{CaO})$, Sodium $\left(\mathrm{Na}_{2} \mathrm{O}\right)$ and phosphorous $\left(\mathrm{P}_{2} \mathrm{O}_{5}\right)$ salts \\
\hline \multirow{4}{*}{3} & \multirow{4}{*}{ After drenching B } & Citrocil $^{\circledR} 0.6 \%$ & 450 ppm IMZ + 600 ppm OPP \\
\hline & & Ortocil $^{\circledR} 0.6 \%$ & 600 ppm OPP \\
\hline & & Citrocide ${ }^{\circledR}$ Plus $0.1 \%$ & 150 ppm PAA + 230 ppm $\mathrm{H}_{2} \mathrm{O}_{2}$ \\
\hline & & Fortisol ${ }^{\circledR} \mathrm{Ca} 1.2 \%$ & Calcium $(\mathrm{CaO})$, Sodium $\left(\mathrm{Na}_{2} \mathrm{O}\right)$ and phosphorous $\left(\mathrm{P}_{2} \mathrm{O}_{5}\right)$ salts \\
\hline 4 & $\begin{array}{l}\text { After drenching } \\
\text { A + washer }\end{array}$ & $\begin{array}{c}(\text { drenching } A)+ \\
\text { Citrocide }{ }^{\circledR} \text { PC 0.5\% }\end{array}$ & $\begin{array}{c}(\text { drenching } A)+ \\
250 p p m \text { PAA + 1150ppm } \mathrm{H}_{2} \mathrm{O}_{2}\end{array}$ \\
\hline 5 & $\begin{array}{l}\text { After drenching } \\
\text { A + washer } \\
\text { + waxing }\end{array}$ & $\begin{array}{c}\text { (drenching A + washer) + Citrosol } \\
\text { Sunseal }{ }^{\circledR} \text { UE IMAD } 2 \text { wax supplemented with Citrotec } 45 \text { SC } \\
1.1 \%+\text { Ortosol } 65001.25 \%\end{array}$ & $\begin{array}{c}\text { (drenching } A+\text { washer) }+ \text { Citrosol Sunseal }^{\circledR} \\
\text { UE IMAD } 2+4950 \text { ppm TBZ }+3575 \text { ppm } \\
\text { OPP }\end{array}$ \\
\hline
\end{tabular}

The two different drenching treatments were applied after harvesting, and the washing and waxing treatments were applied one day afterwards in an industrial packingline. Fruit was sampled as stated. 
amplicons were sequenced using the Sanger methodology. The same forward and reverse primers used for amplification were used for sequencing. In the specific case of the ITS region, the internal primers ITS4 (5'- TCCTCCGCTTATTGATATATGC-3') and LROR (5'- GTACCCGCTGAACTTAAGC3') were also used for the sequencing of the amplicon. Nucleotide sequences were edited with the UGENE program to remove low-quality regions and subsequently assembled [9]. Nucleotide and amino acid sequence comparisons (in the case of EF- $1 \alpha$ and ACT genes) were performed with the MEGA program [10]. Nucleotide and amino acid distances among strains were calculated as p-distances by applying the complete deletion parameter. Multiple alignments were performed using the ClustalW tool available in MEGA [10]. Taxonomic assignment was performed with the Blast tool using the nucleotide collection $\mathrm{nr} / \mathrm{nt}$ database [11].

\section{In vivo infection}

We assayed an in vivo infection with the two C. ramotenellum isolates recovered from affected fruit and identified by genomic sequencing (Isolates 1 and 2, Table 2).

For the in vivo infections W. Murcott mandarins harvested in a Valencia (Spain) area grove were used. Immediately after harvest, fruits were surface disinfected by dipping for 30 seconds in a $0.3 \%$ Citrocide ${ }^{\circledR}$ Plus $(450$ ppm PAA + 690 ppm $\mathrm{H}_{2} \mathrm{O}_{2}$ ) aqueous solution, then randomized and infected.

Two different inoculation methods were used. In the first method, a plastic rod with a 2-mm-long x 0.5-mm-wide steel tip was dipped into a spore suspension and used to make a single wound in each fruit. In the second method, mandarins were immersed in a spore suspension 1 minute.

For both methods, the spore suspension of each isolate was prepared at a concentration of $5 \times 10^{5}$ spores $/ \mathrm{mL}$ from 7 to 10 day old colonies grown on PDA. Conidia were harvested by adding a small amount of sterile distilled water with $0.05 \%$ Tween 20 to each plate and gently rubbing the sporulating mycelial mat with a sterile wire loop. The conidial concentration was adjusted by microscopic counting using a haemocytometer and confirmed by dilution plating. Each inoculation method was assayed twice with both isolates.

\section{Evaluation of fungicide resistance or sensitivity}

To test the sensitivity of the isolates of $C$. ramotenellum to

Table 2: Sequences obtained for the isolates recovered from affected fruit.

\begin{tabular}{|c|c|c|}
\hline Isolate & Gene & Sequence amplified \\
\hline \multirow[b]{3}{*}{1} & ACT & $\begin{array}{l}\text { 5’CGTAAGTCCCAAAACGCCCGCTCTTCTCGCAGCCCGACGGCCAGCTGACAACATCCTAGCTTCCATTG } \\
\text { TCGGCAGACCCCGTCACCATGGGTATGCTCTCCTTGCCCTCCCCACCACCGCCGAATCCAATGTCTAACC-3' }\end{array}$ \\
\hline & EF-1 $\alpha$ & $\begin{array}{l}\text { 5'TCACTTCTTCGCCCCGCCATGACACCCCGCCTCGTCGCAATCAGCGATAAGGCGACACGGCTTGGCTTG } \\
\text { GCGGAGACTTGCTTTCGGTTGAAGACACCACGCCACCATCATCACCCACCTTGTCCACATCACTGACAACC } \\
\text { ATCACAG-3' }\end{array}$ \\
\hline & ITS1-5S-ITS2-28S & $\begin{array}{l}\text { 5’ATAACCCTTTGTTGTCCGACTCTGTTGCCTCCGGGGCGACCCTGCCTTCGGGCGGGGGCTCCGGGTGGA } \\
\text { CACTTCAAACTCTTGCGTAACTTTGCAGTCTGAGTAAACTTAATTAATAAATTAAAACTTTTAACAACGGATCT } \\
\text { CTTGGTTCTGGCATCGATGAAGAACGCAGCGAAATGCGATAAGTAATGTGAATTGCAGAATTCAGTGAATCA } \\
\text { TCGAATCTTTGAACGCACATTGCGCCCCCTGGTATTCCGGGGGGCATGCCTGTTCGAGCGTCATTTCACCA } \\
\text { CTCAAGCCTCGCTTGGTATTGGGCAACGCGGTCCGCCGCGTGCCTCAAACCGTCCGGCTGGGTCTTCTGT } \\
\text { CCCCTAAGCGTTGTGGAAACTATTCGCTAAAGGGTGTTCGGGAGGCTACGCCGTAAAACAACCCCATTTCT } \\
\text { AAGGT-3' }\end{array}$ \\
\hline \multirow[b]{3}{*}{2} & ACT & $\begin{array}{l}\text { 5’ATGTGCAAGGCCGGTTTCGCCGGTGACGATGCGCCCAGAGCCGTTTTCCGTAAGTCCCAAAACGCCCGC } \\
\text { TTCTCTCGGAGCCCGACGGCCAGCTGACAACATCCTAGCTTCCATTGTCGGCAGACCCCGTCACCATGGG } \\
\text { TATGCTCTCCTTGCCCTCCCCACCACCGCCGAATCCAATGTCTAACCGCAGCGCAGTATCATGATCGGTAT } \\
\text { GGGCCAGAAGGACTCGTAATCAATCAATC-3' }\end{array}$ \\
\hline & $E F-1 \alpha$ & $\begin{array}{l}\text { 5'GTCATCGAGAAGTTCGAGAAGGTGAGCACTCTCCCGGCAACTCGCTGTCTTTTCATCGCTGCAATCTCTT } \\
\text { CTTCGCCCCGCCACGACACCCCGCCTCGTCGCAATCCGCGATAAGGCTACAGTGCTTGGCTTGGCGGAGA } \\
\text { CTTGCTCNCGACTGATGACAGCACGCCACCATCAACACCCACCTTGACCACATCACTGACAGTCATCACAG } \\
\text { GAAGCCGCCGAGCTCGGTAAGGGTTCCTTCAAGTAA-3' }\end{array}$ \\
\hline & ITS1-5S-ITS2-28S & $\begin{array}{l}\text { 5’CCCTTTGTACACACCGCCCGTCGCTACTACCGATTGAATGGCTCGGTGAGGCCTTCGGACTGGCCCAGG } \\
\text { GAGGTCGGCAACGACCACCCAGGGCCGGAAAGTTGGTCAAACCCGGTCATTTAGAGGAAGTAAAAGTCGT } \\
\text { AACAAGGTCTCCGTAGGTGAACCTGCGGAGGGATCATTACAAGTGACCCCGGCTACGGCCGGGATGTTCA } \\
\text { TAACCCTTTGTTGTCCGACTCTGTTGCCTCCGGGGCGACCCTGCCTTCGGGCGGGGGCTCCGGGTGGACA } \\
\text { CTTCAAACTCTTGCGTAACTTTGCAGTCTGAGTAAACTTAATTAATAAATTAAAACTTTTAACAACGGATCTCT } \\
\text { ITGGTTCTGGCATCGATGAAGAACGAGCGAAATCGATAAGTAATGTGAATTGCAGAATTCAGTGAATCATC } \\
\text { GAATCTTTGAACGCACATTGCGCCCCCTGGTATTCCGGGGGGCATGCCTGTTCGAGCGTCATTTCACCACT } \\
\text { CAAGCCTCGCTTGGTATTGGGCAACGCGGTCCGCCGCGTGCCTCAAATCGTCCGGCTGGGTCTTCTGTCC } \\
\text { CCTAAGCGTTGTGGAAACTATTCGCTAAAGGGTGTTCGGGAGGCTACGCCGTAAAACAACCCCATTTCTAA } \\
\text { GGTTGACCTCGGATCAGGTAGGGATACCCGCTGAACTTAAGCATATCAATAAGCGGAGGAAAAGAACCAA } \\
\text { CAGGGATTGCTCTAGTAACGGCGAGTGAAGCAGCAATAGCTCAAATTTGAAATCTGGCGTCTTCGACGTCC } \\
\text { GAGTTGTAATTTGTAGAGGATGCTTCTGAGTGGCCACCGACCTAAGTTCCTTGGAACAGGACGTCATAGAG } \\
\text { GGTGAGAATCCCGTATGCGGTCGGAAAGGCGCTCTATACGTAGCTCCTTCGACGAGTCGAGTTGTTTGGG } \\
\text { AATGCAGCTCTAAATGGGAGGTAAATTTCTTCTAAAGCTAAATATTGGCCAGAGACCGATAGCGCACAAGTA } \\
\text { GAGTGATCGAAAGATGAAAAGCACTTTGGAAAGAGAGTTAAAAAGCACGTGAAATTGTTAAAAGGGAAGGG } \\
\text { ATTGCAACCAGACTTGCTCGCGGTGTTCCGCCGGTCTTCTGACCGGTCTACTCGCCGCGTTGCAGGCCAG } \\
\text { CATCGTCTGGTGCCGCTGGATAAGACTTGAGGAATGTAGCTCCCTCGGGAGTGTTATAGCCTCTTGTGATG } \\
\text { CAGCGAGCGCCGGGCGAGGTCCGCGCTTCGGCTAGGATGCTGGCGTAATGGTCGTAATCCGCCCGTCTT } \\
\text { GAAACACGGACCAAGGAGTCTAACATCTATGCGAGTGTTCGGGTGTCAAACCCCTACGCGTAATGAAAGTG } \\
\text { AACGGAGGTGAGAACCGCAAGGTGCATCATCGACCGATCCTGATGTCTTCGGATGGATTTGAGTAAGAGCA } \\
\text { TAGCTGTTGGGACCCGAAAGATGGTGAACTATGCCTGAATAGGGTGAAGCCAGAGGAAACTCTGGTGGAG } \\
\text { GCTCGCAGCGGTTCTGACGTGCAAATCGATCGTCAAATTTGGGTATAGGGGCGAA-3' }\end{array}$ \\
\hline
\end{tabular}


postharvest fungicides, we incubated a spore solution of $1 \times 10^{4}$ spores/mL of each isolate (Isolates 1 and 2, Table 2) in water supplemented with 400 ppm of each fungicide for $18 \mathrm{~h}$. As a control, the isolates were incubated under the same conditions in water without fungicide. The fungicides tested were IMZ, OPP, PCL, PPZ, PYR, and TBZ. After incubation fungicide was removed from the incubation mix by centrifugation at 17000 $\mathrm{g}$ for $10 \mathrm{~min}$ of a $1 \mathrm{~mL}$ sample, the pellet obtained was washed once with sterile water, and then resuspended in $1 \mathrm{~mL}$ of sterile water. Afterwards, aliquots of $100 \mu$ l were plated on triplicate on PDA petri dishes. Plates were incubated at $22{ }^{\circ} \mathrm{C}$ for 2 to 4 days, and the sensitivity/resistance to the corresponding fungicide was evaluated visually. This assay was performed twice with both isolates.

\section{Postharvest treatments}

To test the efficacy of selected postharvest treatments used in the packinghouse to eliminate the inoculum load on the fruit, thus limiting the infection by Cladosporium sp., an assay at industrial level was performed in a packinghouse in Chincha, Perú. Cv W. Murcott mandarins were collected in a field in Chincha and two different postharvest drenching treatments (Table 1) were applied immediately after harvesting. The additional treatments applied in the washer (drenching A + washer) and by waxing (drenching A + washer + waxing) were applied in an industrial packingline the following day. The Cladosporium sp. load on the surface of the fruit was evaluated in 10 fruits randomly selected at different points of the postharvest treatment process, using the method described above for green immature W. Murcott mandarins. Sampling points evaluated were: (1) field fruit without any treatment (as a control to check the basal fungal load); (2) after the drenching treatment A; (4) after the drencher treatment $A$ and washing with Citrocide ${ }^{\circledR}$ PC 0.5\% (drenching $A+$ washer); (5) and after the drencher treatment $A$, the washing with Citrocide ${ }^{\circledR}$ PC $0.5 \%$ and the waxing process with Citrosol Sunseal ${ }^{\circledR}$ UE IMAD 2 (drenching A + washer + waxing). Additionally, we tested the effectiveness of another drenching treatment with a higher dose of OPP (3: drenching treatment B). The treatments applied in each point are described in table 1 .

\section{Statistical analysis}

The assay to compare the effect of the different postharvest treatments on Cladosporium sp. inoculum load on the fruit surface was a one-factor design experiment subjected to analysis of variance (ANOVA) using XLSTAT software ( $v s$. 2019.1.1. Addinsoft Inc, NY, USA). Statistical significance was assessed at the level $p=0.05$, and Fisher multiple range test was used to separate means.

\section{Results and discussion}

\section{Pathogen isolation and identification}

Seven isolates in pure culture were obtained from naturally infected W. Murcott mandarins. The fruit, showing the symptoms of the rot as described, and as showed in figure 1 , were taken from two packinghouses in the Chincha region. After 7 to 10 days at $22^{\circ} \mathrm{C}$ all the isolates grew on PDA as olivaceous to grey-olivaceous colonies due to extensive sporulation. The reverse of the colonies appeared almost black. Microscopically conidiophores of both isolates were pigmented and arose terminally from ascending hyphae. Conidia were slightly curved and globose in shape, pigmented and were produced in branching chains that easily broke apart.

The genetic analysis of the pure cultures of two representative isolates (Isolates 1 and 2) gave the sequences listed in table 2. The sequences obtained were identified by bioinformatics analysis by homology with sequences annotated in the nucleotide collection $\mathrm{nr} / \mathrm{nt}$ database and revealed that the three sequences of both isolates shared 100 $\%$ identity with $C$. ramotenellum.

\section{Cladosporium ramotenellum presence on immature field mandarins}

Cladosporium sp. was recovered in all the analysed fruits taken from the field (fruits coming from a field in Chincha; Figure 1g,h). All the isolates had similar colony and microscopic appearance as the isolates of $C$. ramotenellum obtained from diseased fruit. Three of the field isolates were subjected to genetic identification (Isolates numbered 3 to 5). The sequences obtained are listed in table 3. Again, the analysis revealed that the three sequences of the isolates shared $100 \%$ identity with $C$. ramotenellum.

\section{Fullfillment of Koch's postulates}

To check the ability of the two $C$. ramotenellum isolates recovered from affected fruit (Isolates 1 and 2, Table 2) to cause infection, two different in vivo infection models were tested. In both models the percentage of infection was low; $3 \%-5 \%$ by wound infection, and $5 \%-7 \%$ by immersion. First we used the wound inoculation method and since the percentage of fruit successfully infected was low we tried to inoculate by dipping the fruit in a spore suspension. The inoculated fruit developed the same symptoms that were present in the fruit diseased by natural infection, and, in both cases, we were able to recover both isolates in pure culture from the fruit diseased by in vivo inoculation. The recovered isolates after the infection had the same macroscopic colony characteristics and microscopic conidiophores and conidia morphology as the isolates inoculated.

These results -isolation, identification, and fulfilment of Koch's postulates- establish that this pathogen is the causal agent for the disease described that took place in the Peruvian mandarin shipments in 2018, and again in 2020. The performed isolations and in vivo experiments show that Cladosporium ramotenellum is the causal pathogen of this 
Table 3: Sequences obtained for the isolates recovered from field fruit.

Isolate Gene

ACT

$E F-10$

ITS1-5S-ITS2-

$28 \mathrm{~S}$

ACT

ITS1-5S-ITS2-

$28 \mathrm{~S}$

\section{EF-1 $\alpha$}

EF-1a

ITS1-5S-ITS2-

$28 \mathrm{~S}$
Sequence amplified

5'GTGCAAGGCCGGTTTCGCCGGTGACGATGCGCCCAGAGCCGTTTTCCGTAAGTCCCAAAACGCCCGCTCTTCTCGCAG CCCGACGGCCAGCTGACAACATCCTAGCTTCCATTGTCGGCAGACCCCGTCACCATGGGTATGCTCTCCTTGCCCTCCCC ACCACCGCCGAATCCAATGTCTAACCGCAGCGCAGTATCATGATCGGTATGGGCCAGAAGGACTCGT-3'

5'TCATCGAGAAGTTCGAGAAGGTGAGCACTCTCCCGGCAACTCGTTGTCTTTTCATCGCTGCAATCTCTTCCTCGCCCCGC TATGACACCCCGCCTCGTCGCAATCAGCGATAAGGCGACACGGCTTGGCTTGGCGGAGACTTGCTTTCGGTTGAAGACAC CACGCCACCATCAACACCCTCCTTGACCACATCACTGACAACCATCACAGGAAGCCGCCGAGCTCGGTAAGGGTTCCTTC AAGTAA-3'

5'TGCCCTTTGTACACACCGCCCGTCGCTACTACCGATTGAATGGCTCGGTGAGGCCTTCGGACTGGCCCAGGGAGGTCGG CAACGACCACCCAGGGCCGGAAAGTTGGTCAAACCCGGTCATTTAGAGGAAGTAAAAGTCGTAACAAGGTCTCCGTAGGTG AACCTGCGGAGGGATCATTACAAGTGACCCCGGCTACGGCCGGGATGTTCATAACCCTTTGTTGTCCGACTCTGTTGCCTCC GGGGCGACCCTGCCTTCGGGCGGGGGCTCCGGGTGGACACTTCAAACTCTTGCGTAACTTTGCAGTCTGAGTAAACTTAATT AATAAATTAAAACTTTTAACAACGGATCTCTTGGTTCTGGCATCGATGAAGAACGCAGCGAAATGCGATAAGTAATGTGAATTG CAGAATTCAGTGAATCATCGAATCTTTGAACGCACATTGCGCCCCCTGGTATTCCGGGGGGCATGCCTGTTCGAGCGTCATTT CACCACTCAAGCCTCGCTTGGTATTGGGCAACGCGGTCCGCCGCGTGCCTCAAATCGTCCGGCTGGGTCTTCTGTCCCCTAA GCGTTGTGGAAACTATTCGCTAAAGGGTGTTCGGGAGGCTACGCCGTAAAACAACCCCATTTCTAAGGTTGACCTCGGATCAG GTAGGGATACCCGCTGAACTTAAGCATATCAATAAGCGGAGGAAAAGAAACCAACAGGGATTGCTCTAGTAACGGCGAGTGA AGCAGCAATAGCTCAAATTTGAAATCTGGCGTCTTCGACGTCCGAGTTGTAATTTGTAGAGGATGCTTCTGAGTGGCCACCGA CCTAAGTTCCTTGGAACAGGACGTCATAGAGGGTGAGAATCCCGTATGCGGTCGGAAAGGCGCTCTA

TACGTAGCTCCTTCGACGAGTCGAGTTGTTTGGGAATGCAGCTCTAAATGGGAGGTAAATTTCTTCTAAAGCTAAATATTGGCC AGAGACCGATAGCGCACAAGTAGAGTGATCGAAAGATGAAAAGCACTTTGGAAAGAGAGTTAAAAAGCACGTGAAATTGTTAA AAGGGAAGGGATTGCAACCAGACTTGCTCGCGGTGTTCCGCCGGTCTTCTGACCGGTCTACTCGCCGCGTTGCAGGCCAGC ATCGTCTGGTGCCGCTGGATAAGACTTGAGGAATGTAGCTCCCTCGGGAGTGTTATAGCCTCTTGTGATGCAGCGAGCGCCG GGCGAGGTCCGCGCTTCGGCTAGGATGCTGGCGTAATGGTCGTAATCCGCCCGTCTTGAAACACGGACCAAGGAGTCTAACA TCTATGCGAGTGTTCGGGTGTCAAACCCCTACGCGTAATGAAAGTGAACGGAGGTGAGAACCGCAAGGTGCATCATCGACCG ATCCTGATGTCTTCGGATGGATTTGAGTAAGAGCATAGCTGTTGGGACCCGAAAGATGGTGAACTATGCCTGAATAGGGTGAA GCCAGAGGAAACTCTGGTGGAGGCTCGCAGCGGTTCTGACGTGCAAATCGATCGTCAAATTTGGGTATAGGGGCGAA-3'

5'ATGTGCAAGGCCGGTTTCGCCGGTGACGATGCGCCCAGAGCCGTTTTCCGTAAGTCCCAAAACGCCCGCTCTTCTCGCAGC CCGACGGCCAGCTGACAACATCCTAGCTTCCATTGTCGGCAGACCCCGTCACCATGGGTATGCTCTCCTTGCCCTCCCCACCA CCGCCGAATCCAATGTCTAACCGCAGCGCAGTATCATGATCGGTATGGGCCAGAAGGACTCGTAAAAAA-3'

5'TCATCGAGAAGTTCGAGAAGGTGAGCACTCTCCCGGCAACTCGTTGTCTTTTTCATCGCTGCAATCTCTTCCTCGCCCCGCTA TGACACCCCGCCTCGTCGCAATCAGCGATAAGGCGACACGGCTTGGCTTGGCGGAGACTTGCTTTCGGTTGAAGACACCACG CCACCATCAACACCCTCCTTGACCACATCACTGACAACCATCACAGGAAGCCGCCGAGCTCGGTAAGGGTTCCTTCAAGTAA-3' 5'CCCTTTGTACACACCGCCCGTCGCTACTACCGATTGAATGGCTCGGTGAGGCCTTCGGACTGGCCCAGGGAGGTCGGCAAC GACCACCCAGGGCCGGAAAGTTGGTCAAACCCGGTCATTTAGAGGAAGTAAAAGTCGTAACAAGGTCTCCGTAGGTGAACCTG CGGAGGGATCATTACAAGTGACCCCGGCTACGGCCGGGATGTTCATAACCCTTTGTTGTCCGACTCTGTTGCCTCCGGGGCG ACCCTGCCTTCGGGCGGGGGCTCCGGGTGGACACTTCAAACTCTTGCGTAACTTTGCAGTCTGAGTAAACTTAATTAATAAATT AAAACTTTTAACAACGGATCTCTTGGTTCTGGCATCGATGAAGAACGCAGCGAAATGCGATAAGTAATGTGAATTGCAGAATTC AGTGAATCATCGAATCTTTGAACGCACATTGCGCCCCCTGGTATTCCGGGGGGCATGCCTGTTCGAGCGTCATTTCACCACTC AAGCCTCGCTTGGTATTGGGCAACGCGGTCCGCCGCGTGCCTCAAATCGTCCGGCTGGGTCTTCTGTCCCCTAAGCGTTGTG GAAACTATTCGCTAAAGGGTGTTCGGGAGGCTACGCCGTAAAACAACCCCATTTCTAAGGTTGACCTCGGATCAGGTAGGGAT ACCCGCTGAACTTAAGCATATCAATAAGCGGAGGAAAAGAAACCAACAGGGATTGCTCTAGTAACGGCGAGTGAAGCAGCAAT AGCTCAAATTTGAAATCTGGCGTCTTCGACGTCCGAGTTGTAATTTGTAGAGGATGCTTCTGAGTGGCCACCGACCTAAGTTCC TTGGAACAGGACGTCATAGAGGGTGAGAATCCCGTATGCGGTCGGAAAGGCGCTCTATACGTAGCTCCTTCGACGAGTCGAG TTGTTTGGGAATGCAGCTCTAAATGGGAGGTAAATTTCTTCTAAAGCTAAATATTGGCCAGAGACCGATAGCGCACAAGTAGAG TGATCGAAAGATGAAAAGCACTTTGGAAAGAGAGTTAAAAAGCACGTGAAATTGTTAAAAGGGAAGGGATTGCAACCAGACTTG CTCGCGGTGTTCCGCCGGTCTTCTGACCGGTCTACTCGCCGCGTTGCAGGCCAGCATCGTCTGGTGCCGCTGGATAAGACTT GAGGAATGTAGCTCCCTCGGGAGTGTTATAGCCTCTTGTGATGCAGCGAGCGCCGGGCGAGGTCCGCGCTTCGGCTAGGAT GCTGGCGTAATGGTCGTAATCCGCCCGTCTTGAAACACGGACCAAGGAGTCTAACATCTATGCGAGTGTTCGGGTGTCAAAC CCCTACGCGTAATGAAAGTGAACGGAGGTGAGAACCGCAAGGTGCATCATCGACCGATCCTGATGTCTTCGGATGGATTTGA GTAAGAGCATAGCTGTTGGGACCCGAAAGATGGTGAACTATGCCTGAATAGGGTGAAGCCAGAGGAAACTCTGGTGGAGGC TCGCAGCGGTTCTGACGTGCAAATCGATCGTCAAATTTGGGTATAGGGGCGAA-3'

5'ATGTGCAAGGCCGGTTTCGCCGGTGACGATGCGCCCAGAGCCGTTTTCCGTAAGTCCCAAAACGCCCGCTCTTCTCGCAGC CCGACGGCCAGCTGACAACATCCTAGCTTCCATTGTCGGCAGACCCCGTCACCATGGGTATGCTCTCCTTGCCCTCCCCACC ACCGCCGAATCCAATGTCTAACCGCAGCGCAGTATCATGATCGGTATGGGCCAGAAGGACTCGTAAAAAA-3'

5'GTCATCGAGAAGTTCGAGAAGGTGAGCACTCTCCCGGCAACTCGTTGTCTTTTCATCGCTGCAATCTCTTCCTCGCCCCGCTA TGACACCCCGCCTCGTCGCAATCAGCGATAAGGCGACACGGCTTGGCTTGGCGGAGACTTGCTTTCGGTTGAAGACACCACGC CACCATCAACACCCTCCTTGACCACATCACTGACAACCATCACAGGAAGCCGCCGAGCTCGGTAAGGGTTCCTTCAAGTAA-3' 5'TGCCCTTTGTACACACCGCCCGTCGCTACTACCGATTGAATGGCTCGGTGAGGCCTTCGGACTGGCCCAGGGAGGTCGGCA ACGACCACCCAGGGCCGGAAAGTTGGTCAAACCCGGTCATTTAGAGGAAGTAAAAGTCGTAACAAGGTCTCCGTAGGTGAACC TGCGGAGGGATCATTACAAGTGACCCCGGCTACGGCCGGGATGTTCATAACCCTTTGTTGTCCGACTCTGTTGCCTCCGGGGC GACCCTGCCTTCGGGCGGGGGCTCCGGGTGGACACTTCAAACTCTTGCGTAACTTTGCAGTCTGAGTAAACTTAATTAATAAAT TAAAACTTTTAACAACGGATCTCTTGGTTCTGGCATCGATGAAGAACGCAGCGAAATGCGATAAGTAATGTGAATTGCAGAATTC AGTGAATCATCGAATCTTTGAACGCACATTGCGCCCCCTGGTATTCCGGGGGGCATGCCTGTTCGAGCGTCATTTCACCACTCA AGCCTCGCTTGGTATTGGGCAACGCGGTCCGCCGCGTGCCTCAAATCGTCCGGCTGGGTCTTCTGTCCCCTAAGCGTTGTGGA AACTATTCGCTAAAGGGTGTTCGGGAGGCTACGCCGTAAAACAACCCCATTTCTAAGGTTGACCTCGGATCAGGTAGGGATACC CGCTGAACTTAAGCATATCAATAAGCGGAGGAAAAGAAACCAACAGGGATTGCTCTAGTAACGGCGAGTGAAGCAGCAATAGCT CAAATTTGAAATCTGGCGTCTTCGACGTCCGAGTTGTAATTTGTAGAGGATGCTTCTGAGTGGCCACCGACCTAAGTTCCTTGGA ACAGGACGTCATAGAGGGTGAGAATCCCGTATGCGGTCGGAAAGGCGCTCTATACGTAGCTCCTTCGACGAGTCGAGTTGTTT GGGAATGCAGCTCTAAATGGGAGGTAAATTTCTTCTAAAGCTAAATATTGGCCAGAGACCGATAGCGCACAAGTAGAGTGATCG AAAGATGAAAAGCACTTTGGAAAGAGAGTTAAAAAGCACGTGAAATTGTTAAAAGGGAAGGGATTGCAACCAGACTTGCTCGCG GTGTTCCGCCGGTCTTCTGACCGGTCTACTCGCCGCGTTGCAGGCCAGCATCGTCTGGTGCCGCTGGATAAGACTTGAGGAAT GTAGCTCCCTCGGGAGTGTTATAGCCTCTTGTGATGCAGCGAGCGCCGGGCGAGGTCCGCGCTTCGGCTAGGATGCTGGCG TAATGGTCGTAATCCGCCCGTCTTGAAACACGGACCAAGGAGTCTAACATCTATGCGAGTGTTCGGGTGTCAAACCCCTACGC GTAATGAAAGTGAACGGAGGTGAGAACCGCAAGGTGCATCATCGACCGATCCTGATGTCTTCGGATGGATTTGAGTAAGAGCA TAGCTGTTGGGACCCGAAAGATGGTGAACTATGCCTGAATAGGGTGAAGCCAGAGGAAACTCTGGTGGAGGCTCGCAGCGGT TCTGACGTGCAAATCGATCGTCAAATTTGGGTATAGGGGCGAA-3' 
decay. Since we have established that C. ramotenellum was present on immature fruits in the field, the groves would be the site of the initial contamination of the fruit rind. However, although Cladosporium sp. is reported to be a pathogen for citrus fruit, its incidence is very low when one compares it to the prevalent Penicillium molds, and the stem end rots $[3,12]$. Additionally, since this pathogen requires injuries or weakened areas of the rind for its spores to penetrate [3], one wonders how this pathogen could cause such heavy losses. One hypothesis can be that being citrus fruit Chilling Injury (CI) sensitive, the time and temperatures required for the quarantines have caused the rind damage that has been the gate for the pathogen to penetrate and finally originate the disease. CI is dependent on the binomials time/Temperature, usually more time at a lower Temperature more damage [13] and in Florida, it is well known that grapefruit harvested late in the season is much more sensitive to CI [14]. The fact that late season, and quarantine shipments were more affected is in accordance with the hypothesis that CI damage to a rind contaminated with $C$. ramotenellum spores originated the decay.

Although C. ramotenellum was originally isolated from hypersaline water in Slovenia and belongs to the herbarum species complex [15], and recent studies have shown that it is a very common species found in diverse substrates being widely distributed $[16,17]$, this is the first description of Cladosporium ramotenellum causing decay in citrus. The symptoms of this decay have similarity to the decay symptoms caused by Cladosporium cladosporioides described by Tashiro, et al. [1], so Cladosporium ramotenellum is another Cladosporium species associated with the described sooty spot disease. However, the name "sooty spot" may not be completely appropriate for this disease because resembles to "sooty mold", a well-known citrus cosmetic problem that although is caused by the fungi Capnodium citri, Cladosporium sp., or Meliola sp. [18], can be easily cleaned out from the fruit in the postharvest washing since the affected fruits are not diseased. The similarity between the "sooty spot" and "sooty mold" denominations may bring some confusion to the fresh citrus industry.

\section{Fungicide resistance/sensitivity}

An evaluation of the resistance/sensitivity of the two isolates recovered from affected fruit (isolates 1 and 2, Table 2) to the most-commonly used fungicides applied postharvest on citrus was performed in vitro. The results of these qualitative tests are shown in figure 2. Both isolates were resistant, or mildly resistant, to IMZ, PYR, and TBZ, as an overnight incubation with a $400 \mathrm{ppm}$ solution with these fungicides
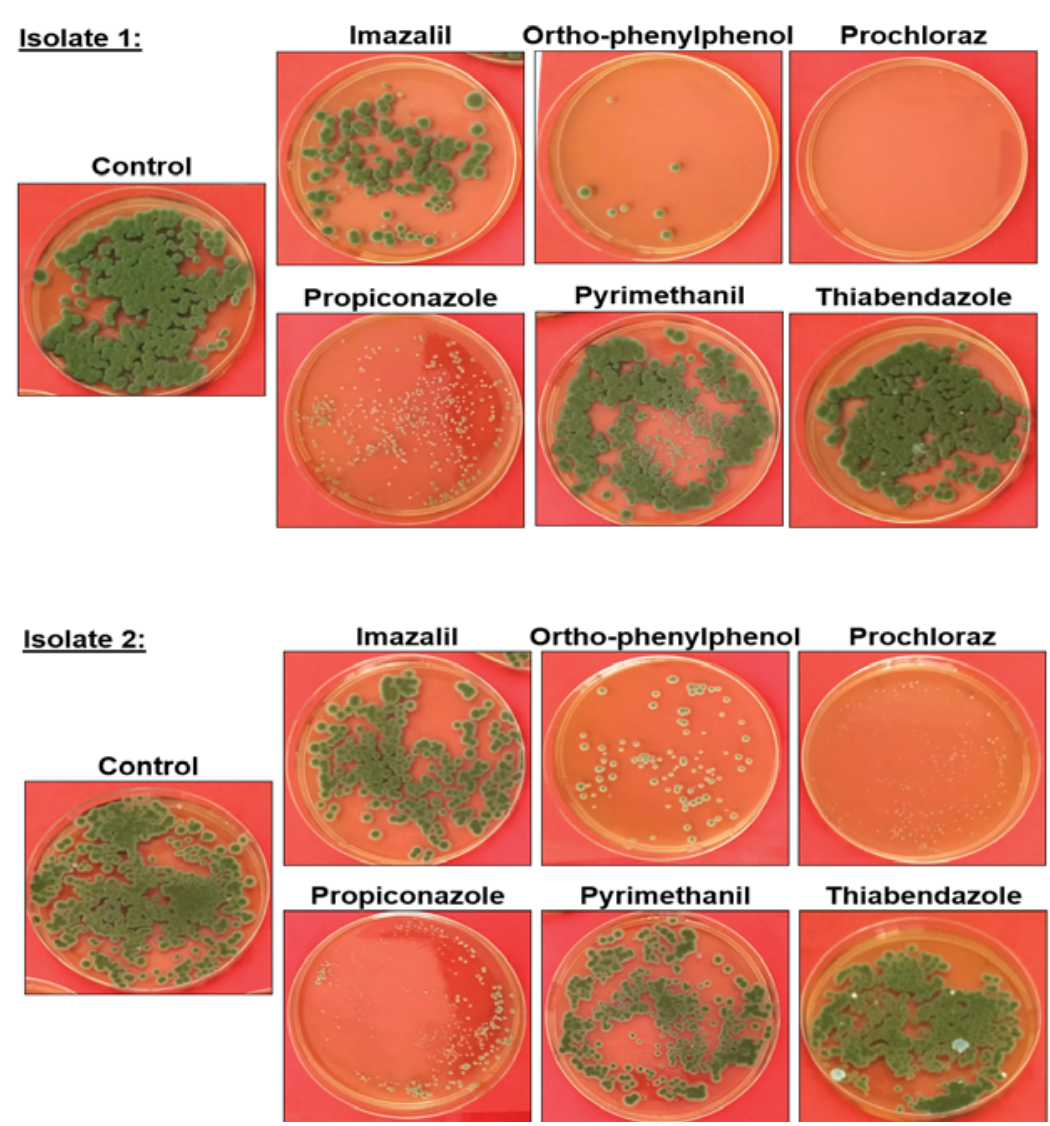

Figure 2: Fungicide resistance/sensitivity of the $C$. ramotenellum isolates recovered from affected fruit. Images show representative PDA agar plates of isolates 1 and 2 grown on PDA after a $18 \mathrm{~h}$ incubation in a $400 \mathrm{ppm}$ solution of the fungicide indicated above the image, or in sterile water (control). Images show one representative plate of the triplicates evaluated in each experiment. 
did not reduced the survival of the isolates. However, the fitness of both isolates was severely impaired when they were incubated with PPZ, OPP, and PCL; some growth was still evident in the plates, but the number of colonies were very low when compared with the control, and the size of the colonies was significantly smaller.

\section{Postharvest treatments reduce spore load and the incidence of the infection}

We checked if some of the postharvest treatments applied in the packinghouse could be useful in reducing the Cladosporium sp. load in the fruit rind. To accurately simulate the fruit postharvest process, we carried out the assays in a packinghouse in the Chincha area (Perú), using its complete processing line, applying the treatments listed in table 1.

As shown in Figure 3, the drenching A treatment reduced by $93,3 \%$ ( $p$ - value $<0.0001$ ) the presence of Cladosporium sp. in the rind. When a higher dose of OPP (1200ppm of OPP in the drenching $B$ treatment) was used in the drencher treatment the reduction was even bigger, a reduction of $97,8 \%$ ( $p$ - value $<0.0001$ ) was achieved.

The postharvest treatments applied after the drenching, the washing performed also with PAA, and the waxing, reduced further the Cladosporium sp. load. After the drenching A treatment, the washing with PAA and the waxing, the reduction was almost complete, a $99,9 \%$ of reduction ( $p$-value $<0.0001$ ) (Figure 3). Additionally, as shown in table 4, after these treatments the presence of Cladosporium sp. was not detectable in some of the fruit analysed; in $50 \%$ of the fruits after the washing treatment, and in $80 \%$ of the fruits

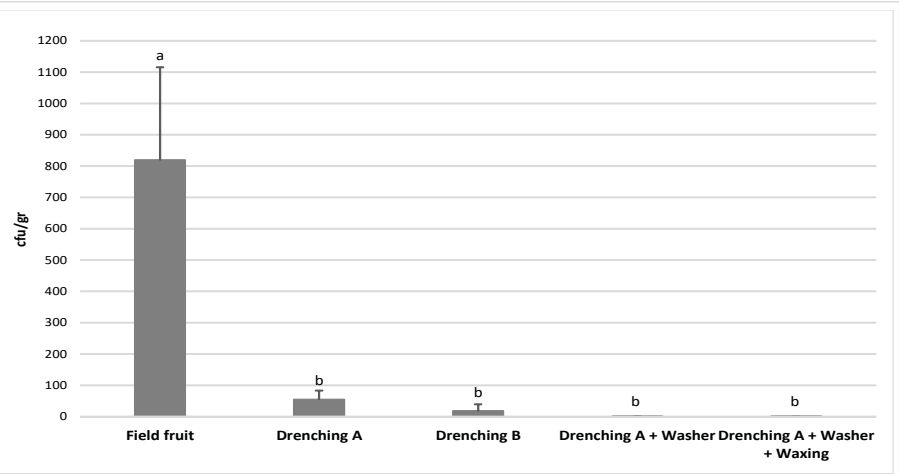

Figure 3: Effect of postharvest treatments on the Cladosporium sp. load on the fruit surface. Cfu/gr was quantified in 10 randomly selected fruits after each postharvest treatment, and it is expressed as the mean number of cfu/gr \pm standard deviation. Different letters denote significant differences $(p<0.05)$ among postharvest treatments.

Table 4: Detection of Cladosporium sp. on the fruit surface after applying the postharvest treatments.

\begin{tabular}{|c|c|}
\hline Postharvest treatment & $\begin{array}{c}\text { Number of fruits with Cladosporium sp. on the } \\
\text { surface } / \mathbf{n}^{\circ} \text { of fruits analysed }\end{array}$ \\
\hline Field fruit/Control & $10 / 10$ \\
\hline Drenching A & $10 / 10$ \\
\hline Drenching B & $9 / 10$ \\
\hline Drenching A + washer & $5 / 10$ \\
\hline $\begin{array}{c}\text { Drenching A + washer + } \\
\text { waxing }\end{array}$ & $2 / 10$ \\
\hline
\end{tabular}

after waxing. These data are of paramount relevance since is difficult to reckon how this disease caused by a Cladosporium species can develop if no spore load of Cladosporium sp. is found in the fruit surface.

The treatments applied in this experiment were the more frequently used in Perú to control the usual citrus decay caused mainly by $P$. digitatum and $P$. italicum, but were supplemented with OPP and two broad spectrum peracetic acid (PAA) disinfectants (Citrocide ${ }^{\circledR}$ Plus in the drenching and Citrocide ${ }^{\circledR}$ PC in the washer). We supplemented the usual postharvest treatment with OPP and PAA because C. ramotenellum is sensitive to both OPP (Figure 2) and PAA (data not shown).

Industrial results of the postharvest treatments applied appeared to be good. Although the pathology didn't appear in the 2019 season it did took place again in 2020, but only in some mandarin shipments from packinghouses that didn't follow these treatments.

Although the contamination and colonization of the fruit by Cladosporium sp. occurs in the field, the treatments tested postharvest limit the extend of the contamination measured both in reduction of $\mathrm{cfu} / \mathrm{gr}$ (Figure 3 ) and in reduction of the percentage of fruit contaminated (Table 4). If confirmed in following seasons that the treatments applied (Table 1) are successful in the control of this decay, this would be another manifestation of the importance of the spore load reduction on the fruit rind to control postharvest diseases. This relationship was clearly established and discussed at length by Eckert and Eaks [19] for P. digitatum and for other pathogens to citrus fruit.

\section{Conclusion}

In summary, we have described and identified for the first time a disease appearing in the long distance shipments of citrus mandarins cv W. Murcott, Nadorcott and Tango. Causal agent is Cladosporium ramotenellum being this proved by application of the Koch postulates. Additionally, we have determined how OPP, PCL and PPZ are effective in vitro in the control of this pathogen. Using this information, and since OPP is, from these three fungicides, the only one whose residues can be legally present on fresh citrus in almost any country of the world [20], we supplemented our usual postharvest treatments for the control of citrus decays with OPP and broad spectrum biocide PAA. The treatment program applied (Table 1) reduced Cladosporium sp. spore load in the fruit rind at least $3 \log$ (Figure 3) and appeared to be successful in the control of the disease.

\section{Acknowledgement}

The authors want to thank Jacqueline Ramallo, Armando Rivadeneyra and Leonor Lazarte from San Miguel Global for providing affected and field fruit samples and for allowing us to use their facilities at Chincha to perform the postharvest treatment assays. Also, we want to thank Rafael Villa-Garcia 
from Fruchincha for providing affected fruit samples, Pedro Cueto from La Calera for providing samples of field fruit and Clodoaldo León from Procitrus for offering his expertise with the pathology and for providing samples of field fruit.

\section{Funding}

This research was entirely funded by Productos Citrosol S.A.; did not receive any other specific grant from funding agencies in the public, commercial, or not-for-profit sectors.

\section{References}

1. Tashiro N, Noguchi M, Ide $\mathrm{Y}$. Sooty spot caused by Cladosporium cladosporioides in postharvest Satsuma mandarin grown in heated greenhouses. J Gen Plant Pathol. 2013; 79: 158-161.

2. Bensch K, Braun U, Groenewald JZ, Crous PW. The genus Cladosporium. Stud Mycol. 2012; 72: 1-401.

PubMed: https://pubmed.ncbi.nlm.nih.gov/22815589/

3. Tuset JJ. Podredumbres de los frutos cítricos. Generalitat Valenciana. Conselleria d'Agricultura i Pesca. 1987.

4. Diaz MA, Vila R. Estudio de la flora fúngica presente en cámaras frigoríficas de conservación de frutos cítricos. Alimentaria. 1987; 183: 77-82.

5. Pascual, J, Blanco, S, Garcia-Lopez, M, García-Salamanca A Bursakov SA, et al. Assessing bacterial diversity in the rhizosphere of Thymus zygis growing in the Sierra Nevada National Park (Spain) through culture-dependent and independent approaches. PLoS One. 2016; 11: e0146558.

PubMed: https://pubmed.ncbi.nlm.nih.gov/26741495/

6. Carbone I, Kohn LM. A method for designing primer sets for speciation studies in filamentous ascomycetes. Mycologia. 1999; 91: 553-556.

7. De Hoog GS, van den Ende AG. Molecular diagnostics of clinical strains of filamentous Basidiomycetes: Molekulare Diagnostik klinischer Stämme filamentöser Basidiomyzeten. Mycoses. 1998; 41: 183-189. PubMed: https://pubmed.ncbi.nlm.nih.gov/9715630/

8. Vilgalys R, Hester M. Rapid genetic identification and mapping of enzymatically amplified ribosomal DNA from several Cryptococcus species. J bacterial. 1990; 172: 4238-4246.

PubMed: https://pubmed.ncbi.nlm.nih.gov/2376561/
9. Okonechnikov K, Golosova O, Fursov M, the UGENE team. Unipro UGENE: a unified bioinformatics toolkit. Bioinformatics. 2012; 28: 1166-1167.

PubMed: https://pubmed.ncbi.nlm.nih.gov/22368248/

10. Kumar S, Stecher G, Li M, Knyaz C, Tamura K. MEGA X: Molecular Evolutionary Genetics Analysis across computing platforms. Mol Biol Evol. 2018; 35: 1547-1549.

PubMed: https://pubmed.ncbi.nlm.nih.gov/29722887/

11. CamachoC,CoulourisG,Avagyan V,MaN,PapadopoulosJ,etal.BLAST+: architecture and applications. BMC Bioinformatics. 2009; 10: 421. PubMed: https://pubmed.ncbi.nlm.nih.gov/20003500/

12. Smilanick JL, Brown GE, Eckert JW. Postharvest citrus diseases and their control. Florida Science Source. Inc. Fresh Citrus Fruits: 2nd Edition. 2006; 339-396.

13. Lafuente MT, Zacarias L. Postharvest physiological disorders in citrus fruit. Stewart Postharvest Review. 2006; 2: 1-9.

14. Purvis AC, Kawada K, Grierson W. Relationship between midseason resistance to chilling injury and reducing sugar level in grapefruit peel. Hort Science. 1979; 14: 227-229.

15. Schubert K, Groenewald JZ, Braun U, Dijksterhuis J, Starink M, et al. Biodiversity in the Cladosporium herbarum complex (Davidiellaceae, Capnodiales), with standardisation of methods for Cladosporium taxonomy and diagnostics. Stud Mycol. 2007; 58: 105-156.

PubMed: https://www.ncbi.nlm.nih.gov/pmc/articles/PMC2104742/

16. Bensch K, Groenewald JZ, Braun U, Dijksterhuis J, Yáñez-Morales $\mathrm{M}$, et al. Common but different: the expanding realm of Cladosporium. Stud Mycol. 2015; 82: 23-74.

PubMed: https://pubmed.ncbi.nlm.nih.gov/26955200/

17. Bensch K, Groenewald JZ, Meijer M, Dijksterhuis J, Jurjević Z, et al. Cladosporium species in indoor environments. Stud Mycol. 2018; 89: 177-301.

PubMed: https://pubmed.ncbi.nlm.nih.gov/29681671/

18. Hall DJ, Sorenson D. Washing, waxing and color-adding. Florida Science Source Inc, Fresh Citrus Fruits: 2nd Edition. 2006; 421-450.

19. Eckert JW, Eaks IL. Postharvest disorders and diseases of citrus fruits. University of California. Division of Natural Resources, Oakland, California. The Citrus Industry. 1989; 4.

20. Zacarias L, Cronje PJR, Palou L. Postharvest technology of citrus fruit. Elsevier Inc. The genus citrus. 2020; 421-446. 